\title{
The aerodynamic forces and pressure distribution of a revolving pigeon wing
}

\author{
James R. Usherwood
}

Received: 9 May 2008/Revised: 11 November 2008/ Accepted: 12 November 2008/Published online: 29 November 2008

(C) The Author(s) 2008. This article is published with open access at Springerlink.com

\begin{abstract}
The aerodynamic forces acting on a revolving dried pigeon wing and a flat card replica were measured with a propeller rig, effectively simulating a wing in continual downstroke. Two methods were adopted: direct measurement of the reaction vertical force and torque via a forceplate, and a map of the pressures along and across the wing measured with differential pressure sensors. Wings were tested at Reynolds numbers up to 108,000, typical for slow-flying pigeons, and considerably above previous similar measurements applied to insect and hummingbird wing and wing models. The pigeon wing out-performed the flat card replica, reaching lift coefficients of 1.64 compared with 1.44. Both real and model wings achieved much higher maximum lift coefficients, and at much higher geometric angles of attack $\left(43^{\circ}\right)$, than would be expected from wings tested in a windtunnel simulating translating flight. It therefore appears that some high-lift mechanisms, possibly analogous to those of slow-flying insects, may be available for birds flapping with wings at high angles of attack. The net magnitude and orientation of aerodynamic forces acting on a revolving pigeon wing can be determined from the differential pressure maps with a moderate degree of precision. With increasing angle of attack, variability in the pressure signals suddenly increases at an angle of attack between $33^{\circ}$ and $38^{\circ}$, close to the angle of highest vertical force coefficient or lift coefficient; stall appears to be delayed compared with measurements from wings in windtunnels.
\end{abstract}

J. R. Usherwood ( $₫)$

Structure and Motion Lab, The Royal Veterinary College,

North Mymms, Hatfield, Herts AL9 7TA, UK

e-mail: jusherwood@rvc.ac.uk

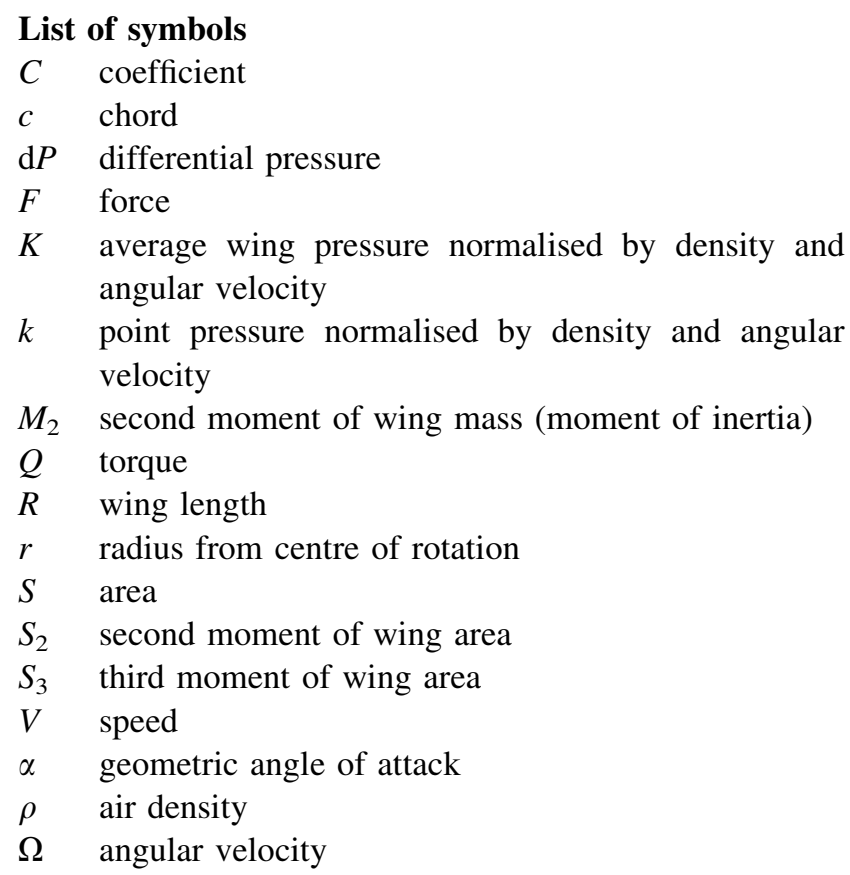

\section{Subscripts}

5areas relating to the average values in each of five areas

5points relating to the values of five sites near the midline

D pro, profile drag

h horizontal

$i \quad$ relating to one of many small wing sections

$j \quad$ relating to one of five sections

L lift

max maximum

$\mathrm{P} \quad$ pressure

$\mathrm{R} \quad$ resultant

v vertical 


\section{Introduction}

The physics of low-speed bird flight is of fundamental aerodynamic, physiological and behavioural interest: do the higher Reynolds numbers experienced by birds preclude insect-like aerodynamics? Exactly how much power does slow, and particularly ascending, flight require from muscle? How do these power requirements relate to foraging and display?

It has recently been suggested that the high force coefficients of flapping insect wings (Ellington et al. 1996; Dickinson et al. 1999; Usherwood and Ellington 2002a, b) may not be maintained at Reynolds numbers appropriate for bird flight, and that hummingbirds might experience some form of aerodynamic transitional regime between insects and the majority of birds (Ellington 2006). Preliminary measurements of a revolving quail wing (Usherwood and Ellington 2002b) at a Reynolds number $R e \approx 26,000$, and those of hummingbird wings (Altshuler et al. 2004) at $R e \approx 5,000$ indicate that lift coefficients above 1.5 can be achieved at angles of attack around $45^{\circ}$. Are these high force coefficients at high angles of attack, reminiscent of those found in flapping or revolving insect wing models, also achieved at higher (though still, in engineering terms, far from high) Reynolds numbers? In this study, a dried wing and a flat card model pigeon wing was tested on a propeller rig (following Usherwood and Ellington 2002a, b; Altshuler et al. 2004), effectively simulating an extended downstroke, at Reynolds numbers up to 108,000 , appropriate for slow, flapping pigeon flight.

Slow, flapping flight, whether during take-off and ascent, hovering or landing, demands a large amount of power from muscle. Indeed, the mass-specific power of quail in ascending flight is currently the highest recorded multi-cycle vertebrate muscle power (Askew et al. 2001). Such high power performances are informative both in terms of muscle physiology (e.g. Askew and Marsh 2001) and behaviour such as display (Usherwood 2008). However, conventional approaches to calculating the power requirements of bird flight (Pennycuick 1975, 1989; Rayner 1979a, b), based on modifications of aeroplane theory, are only valid for medium and fast flight, as profile drag coefficients vary strongly at the high lift coefficients characteristic of low-speed flight (Pennycuick et al. 1992). Modified profile drag coefficients derived from revolving propeller experiments give results, at insect scales at least, equivalent to more sophisticated flapping robot models (though the propeller values can take no account of pronation/supination). Such coefficients can be adopted successfully (e.g. Hedrick and Daniel 2006), but their application into the conventional power calculation framework must be approached with caution.

An alternative approach to calculating the power requirements of flight is to measure the pressures acting along and across the wings (Usherwood et al. 2003, 2005). This sidesteps many of the difficulties inherent in conventional aeroplane- or helicopter-like (blade-element) analyses. Specifically, assumptions need not be made concerning the appropriate lift and drag coefficients, or the influence of the locally induced and unsteady air movements. However, such a technique is reliant on two key assumptions. These are that point differential pressure measurements can be related to the net aerodynamic forces acting on a region, and that the resultant force acts predominantly perpendicular to the local wing chord. In order both to test the validity of these assumptions (and the soundness of the findings of Usherwood et al. 2005) and to provide insight into the local flow behaviour of a revolving pigeon wing, a 16-point pressure map is presented.

\section{Methods}

\subsection{Overview}

The inertial and aerodynamic properties of a dead racing pigeon's wings were measured by slicing and weighing the left wing while fresh, and drying and mounting the right wing on to a DC motor. This formed a one-bladed propeller; the pair of wings from a single bird are mirror images of each other, so cannot form a two-winged, balanced propeller. Slow flight, including take-off, involves relatively low advance ratios: at the midpoint of the first downstroke of a pigeon taking off from a perch (measured at $500 \mathrm{~Hz}$ with the motion analysis system described below) the advance ratio (body speed/wingtip speed) was $0.10 \pm 0.05$ (mean $\pm \mathrm{SD}, N=5$ flights). The propeller setup, therefore, provides a crude simulation of a downstroke during slow or hovering flight. While unsteady, inter- and intra- wing-wing and wing-body aerodynamic interactions are poorly replicated, the propeller system does provide an interesting alternative to the traditional windtunnel measurements, and has the potential to include more realistic three-dimensional aerodynamic effects associated with low advance ratio flapping.

In order to measure the net aerodynamic thrust ( $\approx$ lift) force and torque of the revolving wing, the propeller was mounted on a forceplate. For comparison, the aerodynamic forces acting on a card (3-mm corrugated packing cardboard with edges sealed with insulating tape) model of the dried wing were also measured. Force coefficients for two further pigeon wings are also presented. The aim of this work was to describe one real pigeon wing in as thorough detail as possible; neither cardboard cut-out force nor pressure measurements were taken for the two additional wings. A pressure map of a single, real dried wing was measured by placing a pair of differential pressure transducers through 
the wing feathers. This was repeated eight times, resulting in 16 point pressure measurements distributed along and across the wing.

\subsection{Morphometrics}

A recently killed racing pigeon was dissected, and the wings and flight muscles weighed. The fresh left wing was sliced into 18 strips at 2-cm intervals with a sharp paper guillotine to determine the second moment of wing mass (moment of inertia), $M_{2}$ (see Van Den Berg and Rayner 1995). The right wing was sutured to card board in an outstretched position with the ventral surface flat against the card, and allowed to dry until stiff. This wing was then sutured to a 2.5-mm metal rod, which acted as a sting, connecting the wing to a custom-made aluminium motor head (Fig. 1a). When in situ, mounted on the motor, the dried right wing and its cardboard cut-out replica were photographed, and the relevant wing area moments calculated (Table 1).

The surface topography of each of the three wings was measured with a laser scanner (Polyhemus FastScan, Colchester, Vermont, USA), and are available from the author on request. In order to quantify the degree to which a wing deforms under the aerodynamic and inertial loads of revolving at a range of speeds, reflective tape markers were placed on points on leading and trailing edges and the wingtip (Fig. 1c), and recorded with a high speed $(500 \mathrm{~Hz})$ infra-red 3-D motion analysis system (Qualisys, Gothenburg, Sweden). Deflections are reported at the angle of attack resulting in highest vertical force coefficients.

Fig. 1 Experimental setup of a one-winged propeller formed by a dried or card replica pigeon wing. When mounted on a forceplate $(\mathbf{a}$, here shown at a $+90^{\circ}$ angle of attack), reaction forces indicate the aerodynamic forces acting on the wing as it revolves. Pressure transducers mounted through feather shafts (b) were powered, amplified and logged by a unit revolving with the propeller head. Eight repeats (two sites each time) result in a pressure map of 16 sites (c) along the wing. Black circles indicate sites near the midline of the wing. Stars indicate later positioning of motion analysis markers; the symbols underneath the wing relate to wing positions from base to tip, matching the symbols in Fig. 2b

\subsection{Propeller and forceplate setup}

The 2.5-mm rod connecting the dried wing, or the flat card cut-out wing, was connected through the centre of 72-tooth gear cog (Fig. 1a). This allowed precise $5^{\circ}$ increments in wing angle, and also ensured that the wing was held at a consistent distance from the propeller head throughout the range of geometric angles of attack. The centre of rotation was $34 \mathrm{~mm}$ from the humerus, allowing the full range of angles of attack to be measured. This, in effect, reduced the geometric advance ratio from 0 to 0.09 . While having some aerodynamic effect, this is likely to be minor (see Dickson and Dickinson 2004). An initial geometric angle of attack was measured in situ at the wing tip marked in Fig. 1 using a digital inclinometer. This site is convenient because it is relatively flat and thin, and relevant because this portion of the wing moves relatively fast, producing disproportionately high aerodynamic forces. A counter-balance mass was connected to the same rod on the other side of the propeller head. The $12-\mathrm{V}$ high-torque DC motor was mounted vertically in a 1-m card tube, which was mounted directly on to a Kistler 9287B forceplate, and stabilised by four tensioning wires running to each corner of the forceplate (Fig. 1b). The wing was mounted such that the 'thrust', 'weight-supporting' or 'lifting' force was orientated directly downwards; the propeller downwash was directed upwards. The ceiling and walls were at least $2.5 \mathrm{~m}$ from the wing; aerodynamic wall- and ground-effects were considered negligible. The effect of in-wash to the propeller at the level of the forceplate was minimal; propellerinduced drafts did not affect a small down feather placed
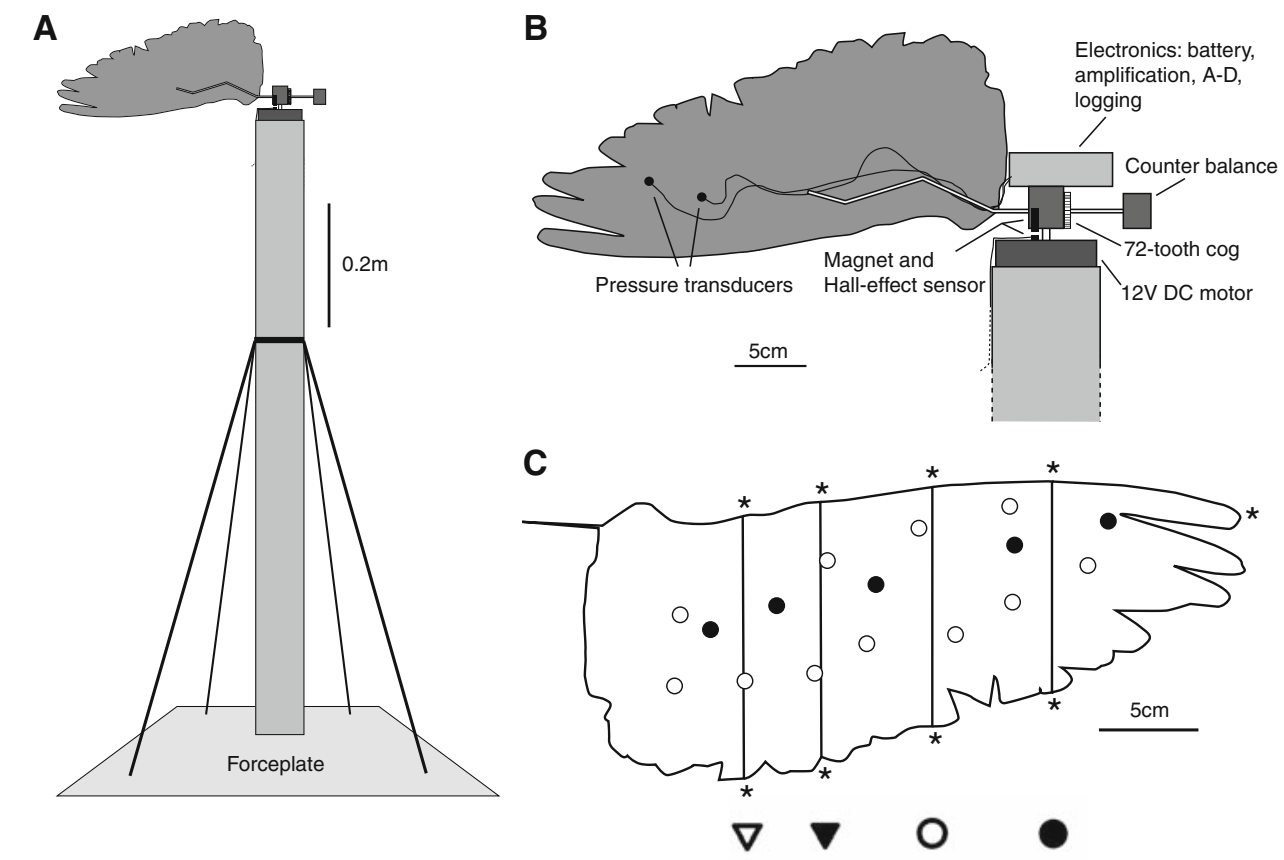
Table 1 Relevant morphometrics of the dried right pigeon wing and its cardboard model calculated from photographs taken once mounted on to the motor

\begin{tabular}{lllll}
\hline & Right wing & Right wing on sting & Card wing & Card wing on sting \\
\hline$R(\mathrm{~m})$ & 0.326 & 0.364 & 0.343 & 0.375 \\
$S\left(\mathrm{~m}^{2}\right)$ & $3.22 \times 10^{-2}$ & $3.22 \times 10^{-2}$ & $3.66 \times 10^{-2}$ & $3.66 \times 10^{-2}$ \\
$S_{2}\left(\mathrm{~m}^{4}\right)$ & $9.53 \times 10^{-4}$ & $1.42 \times 10^{-3}$ & $1.13 \times 10^{-3}$ & $1.57 \times 10^{-3}$ \\
$S_{3}\left(\mathrm{~m}^{5}\right)$ & $2.09 \times 10^{-4}$ & $3.52 \times 10^{-4}$ & $2.61 \times 10^{-4}$ & $4.03 \times 10^{-4}$ \\
\hline
\end{tabular}

on the forceplate, so cowling of the forceplate was considered unnecessary.

In order to measure rotational frequency, a small bar magnet was mounted on the propeller head, which triggered a Hall-effect sensor mounted on the top of the propeller body once a cycle. In order to drive the propeller at set speeds despite radically differing aerodynamic loads, this magnetometer signal was measured and used to determine the power output of a power supply (N5743A, Agilent Technologies, Wokingham, Berks, UK) [analogue in and out signals were received/emitted at $500 \mathrm{~Hz}$ through a National Instruments (Austin, TX) 6024E PCMCIA card]. Actual measured rotational frequencies for each trial were used in subsequent calculations; while they differed only slightly from the intended frequencies (coefficient of variation $<2 \%$ ), aerodynamic forces are broadly proportional to the square of velocity, so it is worth using the most accurate measure of rotational frequency in calculating coefficients.

\subsection{Reynolds number}

The chord-based Reynolds number (using Ellington's (1984c) formulation) for a pigeon in slow flight (Usherwood et al. 2005) is of the order of 92,000 [wing span of $0.302 \mathrm{~m}$, wing area $0.0296 \mathrm{~m}^{2}$, wingbeat frequency $7.98 \mathrm{~Hz}$ (downstroke period $0.0673 \mathrm{~s}$ ), downstroke amplitude approximately $180^{\circ}$ ] and the aerodynamic power derived from direct pressure measurements was $12.8 \mathrm{~W}$ (single wing). Therefore, a rotational frequency of $8 \mathrm{~Hz}$ for a $12 \mathrm{~V}, 7.2$-A motor provides a suitable comparison to the slow-flying pigeon (providing $R e \approx 108,000$ ). However, at high angles of attack-potentially of relevance during take-off, hovering and landing - the power demands on the motor operating at $8 \mathrm{~Hz}$ were excessive. Therefore, to provide aerodynamic coefficients covering the complete range of potentially relevant angles of attack, the propeller was also driven at $4 \mathrm{~Hz}(\operatorname{Re} \approx 54,000$, a quarter of the aerodynamic forces and an eighth of the power requirements). At extreme angles of attack $\left(90^{\circ} \pm 10^{\circ}\right)$, the power requirements at even $4 \mathrm{~Hz}$ were too much for the motor, and the rotational frequency was further reduced, down to $3.6 \mathrm{~Hz}(\operatorname{Re} \approx 49,000)$.
2.5 Presentation of forceplate results

Real dried and model card wings were tested at $8 \mathrm{~Hz}$ (for 10 angles around zero angle of attack) and at 4-3.6 Hz (for 24 angles, ranging from below zero to above $90^{\circ}$ ). The reaction forces measured by the forceplate at $500 \mathrm{~Hz}$ were used to determine aerodynamic forces. The thrust force $F_{v}$, coaxial with the propeller, acted vertically. Using the blade-element analysis (e.g. Osborne 1951; Weis-Fogh 1973; Ellington 1984a, b, c; Usherwood and Ellington 2002a) for revolving wings, a mean vertical force coefficient $C_{\mathrm{v}}$ can be derived, as

$F_{\mathrm{v}}=\int_{0}^{R} \frac{\rho}{2} C_{\mathrm{v}, i} c_{i} V_{i}^{2} \mathrm{~d} r=\frac{\rho}{2} C_{\mathrm{v}} \Omega^{2} S_{2}$,

where $\rho$ is the density of air (taken as $1.2 \mathrm{~kg} / \mathrm{m}^{3}$ ), $C_{\mathrm{v}, i}$ the vertical force coefficient, $c_{i}$ the chord and $V_{i}$ the velocity, each for element $i$ along the entire length $(R)$ of the wing. $\Omega$ is the angular velocity, and $S_{2}$ the second moment of a single wing's area. Thus,

$C_{\mathrm{v}}=\frac{2 F_{\mathrm{v}}}{\Omega^{2} S_{2}}$

While variations in horizontal reaction forces experienced by the forceplate could, in theory, be used to determine the average aerodynamic drag and position of the centre of drag directly, this requires a very high frequency response: mechanical smoothing of forces transmitted between the wing and the forceplate would result in an under-calculation of drag (as mean horizontal force experienced by the forceplate is zero) and miss-calculation of the centre of drag towards the centre of rotation. Instead, drag is derived from the net torque $(Q)$ measurements about the vertical axis following the standard blade-element formulation:

$Q=\int_{0}^{R} \frac{\rho}{2} C_{\mathrm{h}, i} c_{i} r_{i} V_{i}^{2} d r=\frac{\rho}{2} C_{\mathrm{h}} \Omega^{2} S_{3}$,

where $C_{\mathrm{h}}$ is the mean horizontal force coefficient taken to act across the wing, and $S_{3}$ is the third moment of a single wing's area. Thus, 
$C_{\mathrm{h}}=\frac{2 Q}{\Omega^{2} S_{3}}$.

Note that, in this study, a distinction is made between horizontal and vertical force coefficients, and drag and lift coefficients. Aerodynamic drag and lift coefficients relate to forces parallel and perpendicular to the airflow, respectively; in the case of a static but revolving propeller, the induced flow at the blade (or wing) may be considerable. Drag and lift coefficients are calculated using the large-angle formulation of Usherwood and Ellington (2002a), which takes account of the changes in geometry associated with a downwash calculated as a conventional Rankine-Froude momentum jet and a triangular downwash distribution. However, the effect to the main points of this study of the distinction between $C_{\mathrm{h}}$ and $C_{D \text {,pro }}$, and $C_{\mathrm{v}}$ and $C_{\mathrm{L}}$ is minor. This blade-element approach is based on the 2-D assumption that the aerodynamic consequences (lift, drag) of conditions at each element (angle of attack, velocity, chord width and profile) can be combined as a simple summation; aerodynamic interaction between elements is not accounted for. However, using this terminology even for cases where strong spanwise flows are suspected allows comparisons between wing properties to be made despite very different motions (translating, rotating, flapping, etc.), and indeed can indicate where 3-D flows are likely to be important.

\subsection{Pressure measurements}

Differential pressures between lower and upper wing surfaces were measured at 16 sites ( 8 sets of experiments, 2 pressure sites each set) across the wing operating at $4 \mathrm{~Hz}$ (down to $3.6 \mathrm{~Hz}$ as before), over 24 angles of attack ranging from $-17^{\circ}$ to $+98^{\circ}$ at the wingtip. Hollowed pen nibs glued over the gauge hole of the pressure sensors (EPE-EO1-2P, Measurement Specialties, Hampton, VA USA) were pushed through feather shafts and, where necessary, held in place by hot glue (see Usherwood et al. 2005). At central portions of the wing, covert feathers were prevented from obstructing the ports by the application of a small tab of cloth tape. The nibs projected from the upper wing surface by approximately $1 \%$ of wing chord width. The pressure sensors and op-amps were powered from the stabilised supply provided by the data logger (Logomatic, Sparkfun Electronics, Boulder, CO, USA). Pressure signals were logged at 10 bits at $500 \mathrm{~Hz}$ per channel and written to an SD card. Each pressure sensor was calibrated directly after the experiments by raising a column of water in 5-mm intervals. This was achieved by pushing the pen nib through a latex glove tightly stretched over a stiff, vertical tube filled with water, with the open end of the tube in water.

The pressure sensors have a rated frequency response within $\pm 1 / 2 \mathrm{~dB}$ to $5 \mathrm{kHz}$, so sampling at $0.5 \mathrm{kHz}$ allows recording of real aerodynamic phenomena; signal variability may have some value, potentially indicating the chaotic flow usually associated with stall.

Results from pressure measurements are presented in two ways. The first uses the coefficient of pressure $C_{\mathrm{P}}$, formulated to be directly comparable with the propeller force coefficients:

$\frac{\text { Aerodynamic force }}{\text { area }}=\mathrm{d} P=\frac{\rho}{2} C_{\mathrm{P}} V^{2}$

where $\mathrm{d} P$ is the differential pressure measured at a point and $V$ the velocity at that point. So, for a revolving wing, and a pressure site at distance $r$ from the propeller axis,

$C_{\mathrm{P}}=\frac{2 \mathrm{~d} P}{\rho \Omega^{2} r^{2}}$.

The second way of presenting the data keeps the relative magnitudes of the various sites, maintaining the relative importance of outer (faster) wing sections. In this case, the term $r$ is removed:

$k_{\mathrm{p}}=\frac{2 \mathrm{~d} P}{\rho \Omega^{2}}$

This, taken over the whole wing using a resultant force coefficient $\left(C_{\mathrm{R}}=\sqrt{C_{\mathrm{h}}^{2}+C_{\mathrm{v}}^{2}}\right)$, is equivalent to

$K_{\mathrm{p}}=C_{\mathrm{R}} \frac{S_{2}}{S}$

from the forceplate measurements.

\subsection{Signal analysis}

Each test, for each wing, angle of attack, pressure positioning or forceplate measurement, started with the wing at rest for $9 \mathrm{~s}$, followed by an impulsive start up to the required rotational frequency, which was maintained for $4 \mathrm{~s}$. After this the propeller came to a halt for $7 \mathrm{~s}$, and started and stopped two further times. For both forceplate and pressure measurements, a linear drift was removed from just before to just after each period of rotation. The values from $N-10$ to $N-2$ (where $N=0$ is the last revolution), as indicated by the magnetometer, were assessed. This period was after the downwash had fully developed, and before the wing had started to slow. The average rotational frequency, vertical force and torque or differential pressures for these three periods were calculated. For the pressure measurements, the standard deviation during each of the three periods was calculated and averaged. 
2.8 Derivation of coefficients from differential pressure measurements

The areas of five regions (Fig. 1C) down the wing were measured from photograph. Also, the twist of the chord relative to the wingtip chord was measured with a digital inclinometer so that the geometric angle of attack could be determined for each section at each set angle of attack. The average resultant force coefficient derived from the differential pressure measurements was derived in two ways: first, the average differential pressure for all of the sensors within an area was multiplied by the section area to provide the section force; second, only those pressure sensors near the midline of the wing (Fig. 1C) were taken as representative for the pressure across the whole wing section. The purpose of the midline-only contributions was to determine whether a reduced set of measurements-far more realisable with free-flying, live, flapping birds-might be sufficient to provide informative values. The contribution of each section $j$ to the resultant aerodynamic force $F_{R, j}$ is

$F_{\mathrm{R}, j}=\mathrm{d} P_{j} S_{j}$,

where $\mathrm{d} P_{j}$ is the representative differential pressure for the section (either the average or that of the mid-line sensor) and $S_{j}$ the area of the section. If this resultant aerodynamic force acts perpendicular to the wing section-an assumption shown to be broadly valid for model insect wings at high angles of attack (Dickinson 1996; Dickinson et al. 1999; Usherwood and Ellington 2002a), and only likely to be incorrect by a few degrees for fully attached flow with leading-edge suction (typical of low angles of attack) - then the contribution of each section to horizontal and vertical force coefficients is a simple matter of geometry:

$C_{\mathrm{h}}=\frac{2}{\rho \Omega^{2} S_{2}} \sum F_{\mathrm{R}, j} \sin \left(\alpha_{j}\right)$,

and
$C_{\mathrm{v}}=\frac{2}{\rho \Omega^{2} S_{2}} \sum F_{\mathrm{R}, j} \cos \left(\alpha_{j}\right)$

where $\alpha_{j}$ is the geometric angle of attack of section $j$.

\section{Results and discussion}

\subsection{Morphometrics}

The racing pigeon had the following morphometry: mass, $507.4 \mathrm{~g}$; total pectoralis, $149.7 \mathrm{~g}$; total supracoracoideous, $16.1 \mathrm{~g}$; total wing mass, $73.2 \mathrm{~g}$. The left wing, while still fresh, had a second moment of mass of $3.28 \times$ $10^{-4} \mathrm{~kg} \mathrm{~m}^{2}$. The wing lengths, areas, and moments of areas for the dried right wing and the cardboard model are shown in Table 1.

High speed motion analysis of this wing revolving at the angle of attack resulting in the maximum vertical force coefficient, at a range of rotational frequencies, demonstrates that wings can undergo considerable passive deflections due to life-like aerodynamic forces (Fig. 2): the wing tip deflected 'upwards' (in the aerodynamic sense) by around $100 \mathrm{~mm}$; the angle of attack decreased towards the wing base, but increased slightly at the wingtip.

\subsection{Force coefficients}

Force coefficients for stiff, flat model pigeon wings operating at $R e \approx 108,000$ and 54,000 match those of simple hawkmoth wing models operating at $R e \approx 8,000$ (Fig. 3), which have been shown to be largely indistinguishableexcept for a lesser minimum drag coefficient-from Drosophila wings operating at $R e \approx 200$ (Dickinson et al. 1999; Usherwood and Ellington 2002b). It is therefore difficult to support the notion of a critical Reynolds number above which the high-lift mechanisms of revolving insect wings fail (see Ellington 2006). Whether the
Fig. 2 Deflections of wing tips 'upwards' (in the aerodynamic sense) (a) and wing sections, influencing the angles of attack (b), for a dried pigeon wing for a range of rotational frequencies, at the angle of attack resulting in the highest vertical force coefficients (initially set at $38^{\circ}$ )
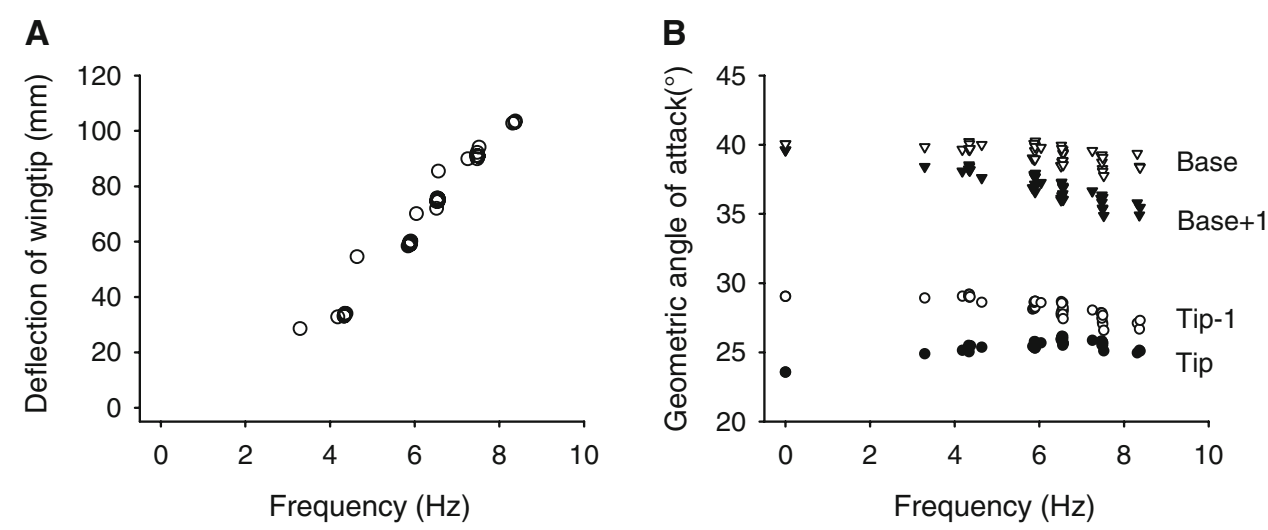

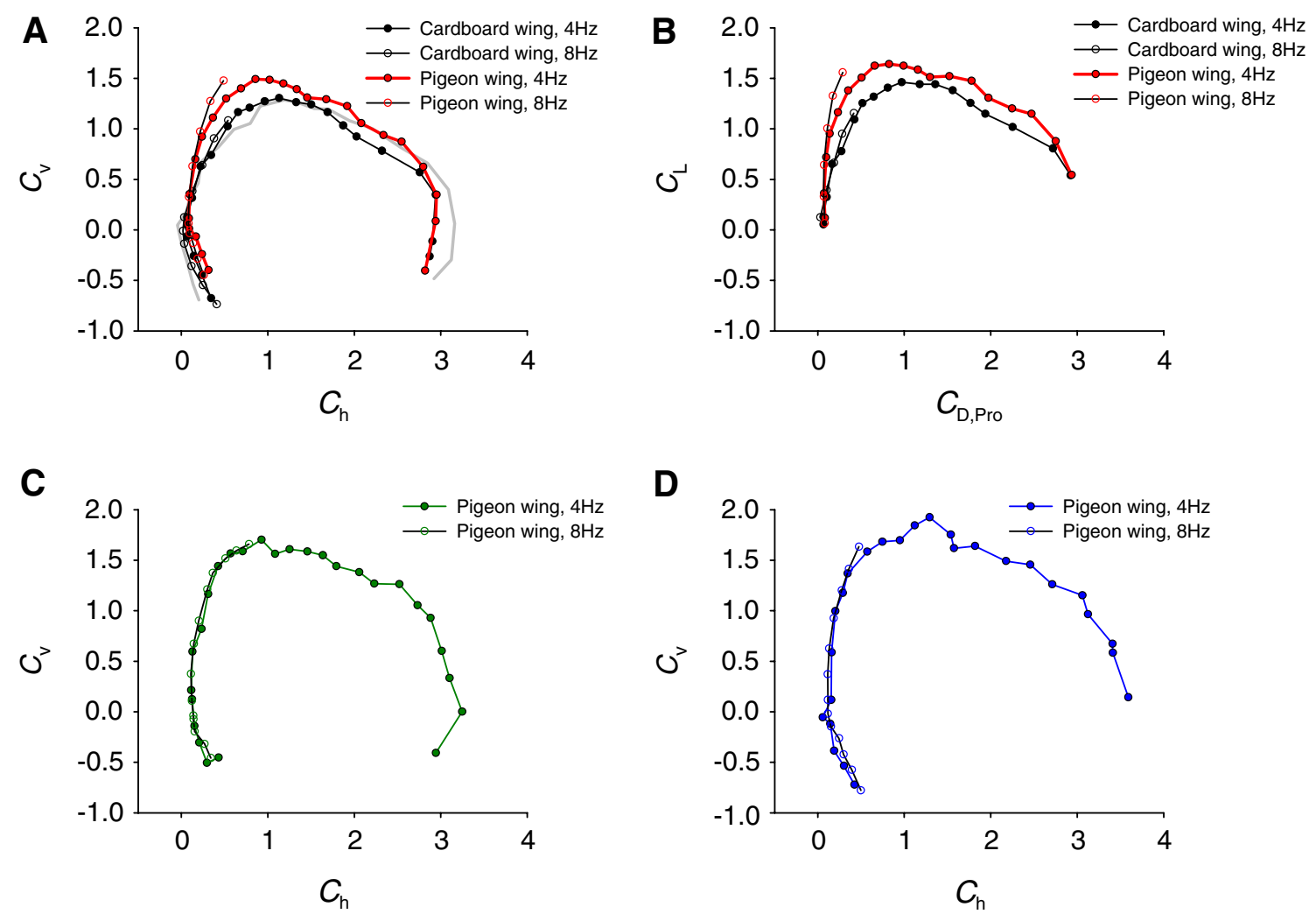

Fig. 3 Aerodynamic force coefficients for revolving real, dried (coloured, a-d) and flat card (blacklines, black circles) pigeon wings. Open circles indicate values with a rotational frequency of $8 \mathrm{~Hz}$ $(R e \approx 108,000)$; filled circles at approximately $4 \mathrm{~Hz}(R e \approx 54,000)$. The underlying grey plot (a) indicates values from flat model

hawkmoth wings at $R e \approx 8,100$ from Usherwood and Ellington 2002b. Horizontal and vertical force coefficients (from a) are transformed to lift and profile drag coefficients (b) avoiding small angle assumptions following Usherwood and Ellington 2002a. c and d show force coefficients for two further pigeon wings

aerodynamic mechanisms behind the high forces are identical is doubtful; whether they are broadly similar remains controversial (is spanwise flow present or required at all Reynolds numbers?), but such issues motivate future DPIV investigations.

The real, dried wing performs considerably better than the card replica wing (Fig. 3a, b), with the maximum lift coefficient for the dried wing of 1.64, and for its card replica of 1.44. Whereas attempts at improving upon flatwinged performance of model hawkmoth wings by introducing camber and twist largely failed (Usherwood and Ellington 2002a), some attributes of the real bird wing clearly makes a notable difference. The salient differences between the flat, card wing and the real, dried pigeon wing is not yet clear. Therefore, an attractive line of future research to tease apart which factors contribute to this difference, parallel to that of Ellington's for hummingbird wings, would involve: imaging and reconstructing the wing, varying the aerofoil and whole-wing properties, and rapid prototyping and aerodynamic propeller-testing.

In the case of the main real, dried wing of this study, but much less so (if at all) the other dried wings or the card replica, vertical or lift coefficient increases far more rapidly

with angle of attack at high rotational frequencies than low (Fig. 3). While an increase in maximum vertical or lift coefficients are not observed (potentially because the motor was unable to power the wing at sufficiently high angles of attack at $8 \mathrm{~Hz}$ ), performance is improved at high speeds as the high lifts occur at relatively low drags: $C_{\mathrm{L}} / C_{\mathrm{D} \text {,pro }}$ at maximum lift coefficient were 5.4 and 2.0 for fast and slower real wings, respectively. One account for this phenomenon, and possibly its inconsistency between real wings and absence in the card wing, might be aeroelastic deflection. The pigeon wings were dried fully outstretched, tied with their ventral surfaces flattened; this somewhat arbitrary and inconsistent wing form may benefit from the deflections, particularly the twist, imposed by aerodynamic (and, potentially, 'centrifugal' inertial) loads (Fig. 2).

Lift coefficients, derived by transforming horizontal and vertical force coefficients to take account of the flow induced at the level of the wing, are, as expected, somewhat higher than $C_{\mathrm{v}}$ (apparently contradicting Altshuler et al. 2004); the performance of the wing would be somewhat higher for the first flap of take-off, before the wake had fully formed. Whether considering $C_{\mathrm{L}}$, which requires a range of simplifying assumptions, or accepting 

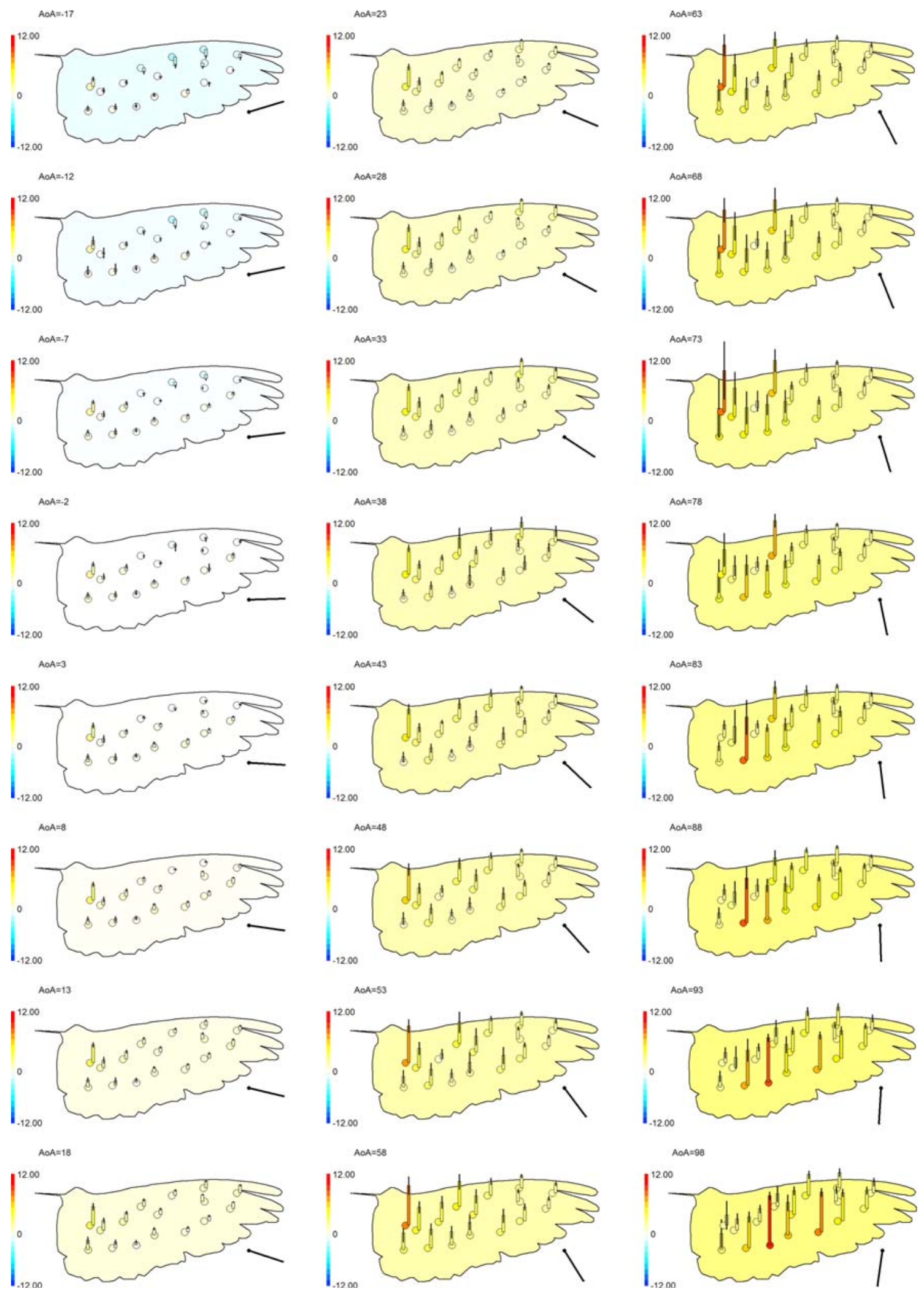

Fig. 4 Coefficients of pressure $C_{\mathrm{P}}$ from point pressure measurements, and for the whole wing derived from forceplate measurements (background colours). Black vertical bars show $\pm 6 \mathrm{SD}$ of the pressure-derived signals. The scale bars to the left relate pressure coefficients to both colour and column (and error bar) height 

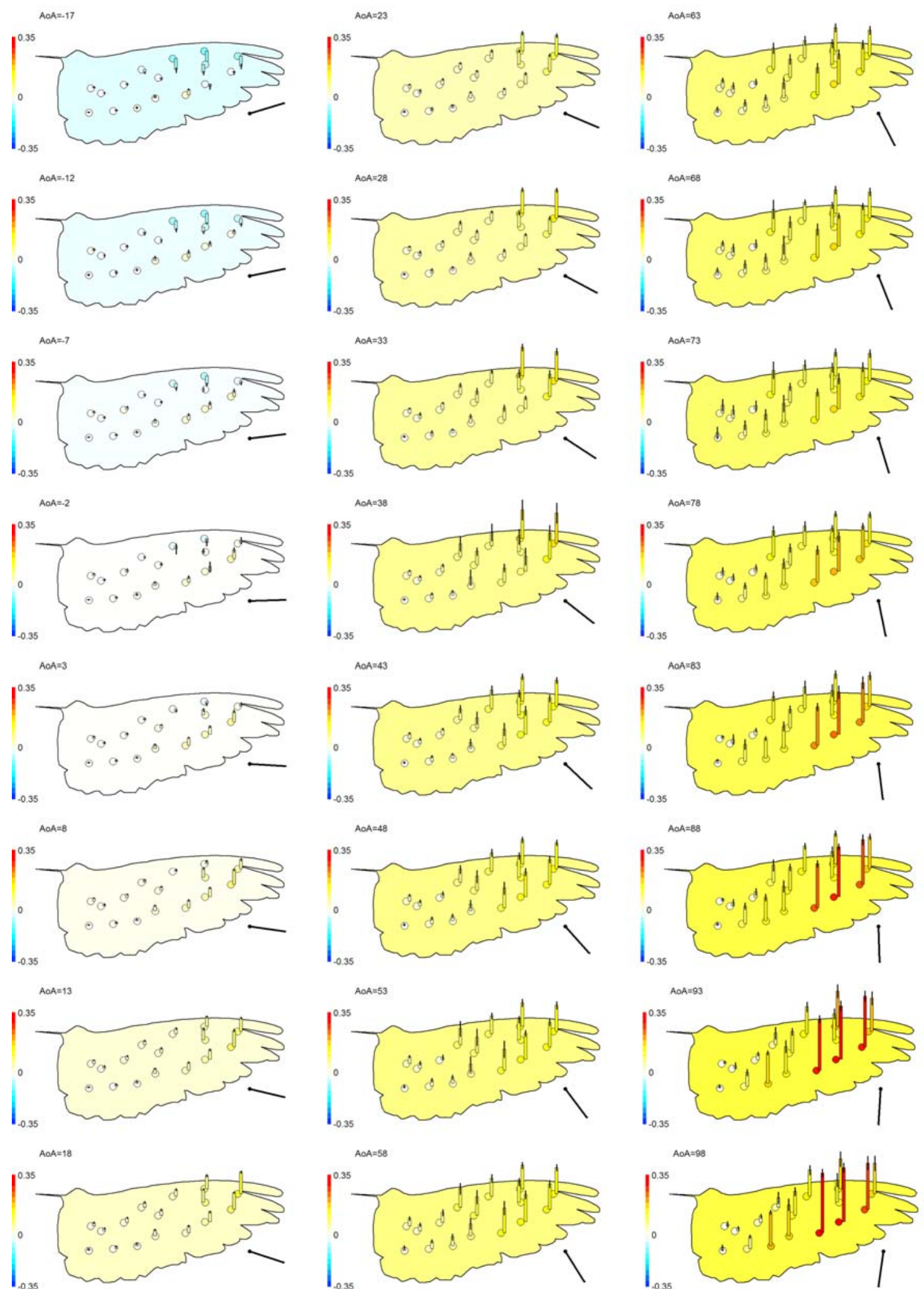

Fig. 5 Point $\left(k_{\mathrm{p}}\right)$ and whole wing $\left(K_{\mathrm{p}}\right.$, background wing colours $)$ differential pressures normalised by air density and the square of angular velocity, but not (unlike the coefficients in Fig. 4) by distance from the centre of rotation. Therefore, values show true relative magnitudes: larger differential pressures occur at the (faster moving) wingtip. Black vertical bars show $\pm 6 \mathrm{SD}$ of the pressure-derived signals. The scale bars to the left relate pressure coefficients to both colour and column (and error bar) height 
Fig. 6 Force coefficients derived from forceplate measurements (bold black), and differential pressure measurements (red), using averages of all the pressure measurements for each section, and assuming the resultant aerodynamic force acts perpendicular to each wing chord. Blue lines bounding the pressure-derived values show coefficients derived with $\pm 2 \mathrm{SD}$ of the measured pressure signals. The vertical dashed lines indicate the angle at which stall is postulated

Fig. 7 Force coefficients derived from forceplate measurements (bold black), and differential pressure

measurements (red), using only mid-line pressure measurements for each section (in contrast to Fig. 6) indicated in Fig. 1c. Blue lines bounding the pressure-derived values show coefficients derived with \pm 2 SD of the measured pressure signals
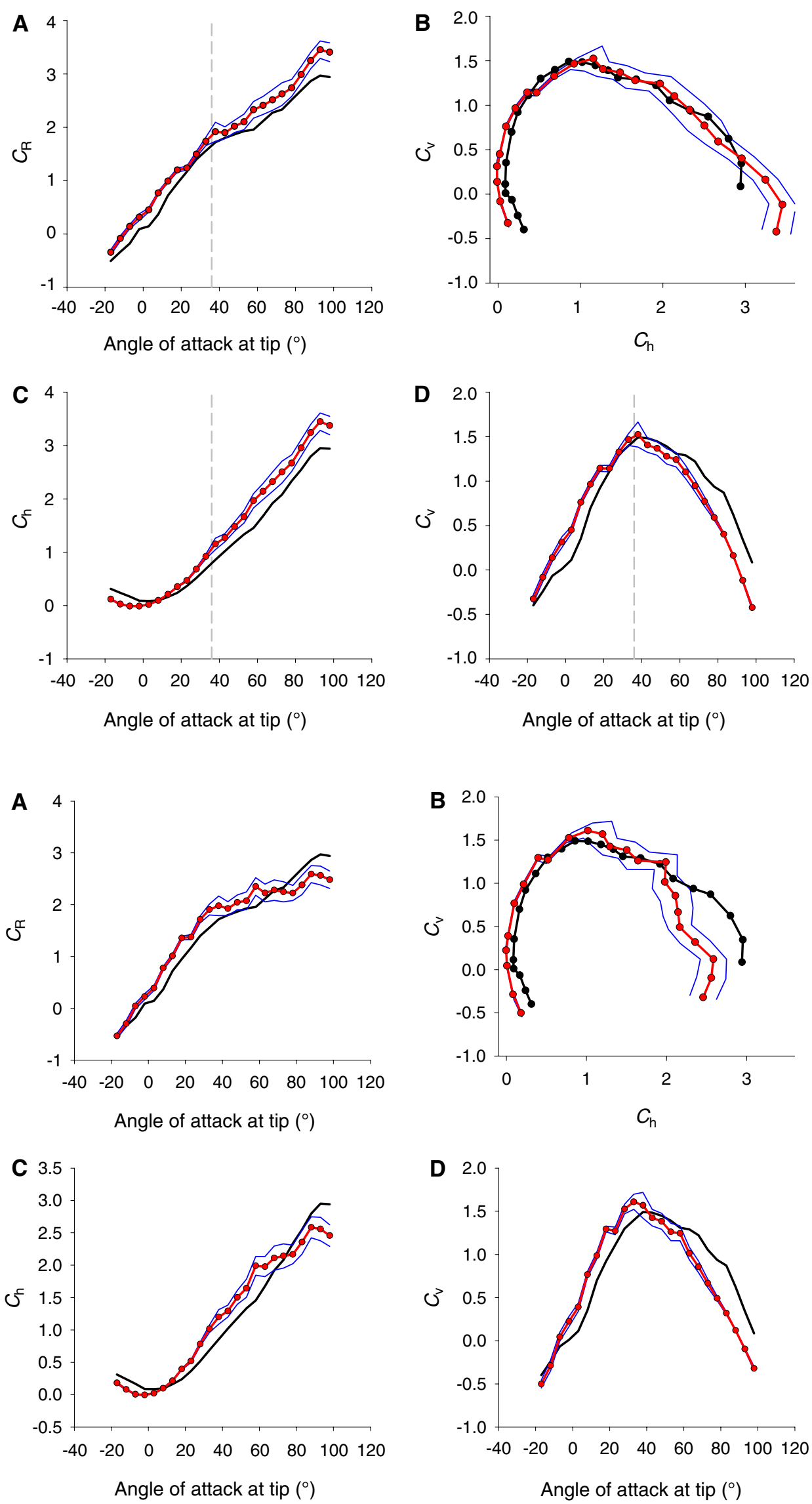
Table 2 Linear regression coefficients for resultant, horizontal and vertical force coefficients $\left(C_{\mathrm{R}}, C_{\mathrm{h}}\right.$ and $\left.C_{\mathrm{v}}\right)$ derived from forceplate measurements (subscript FP) predicted from pressure measurements across five areas (subscript 5areas) or five points along the wing midline (subscript 5points)

\begin{tabular}{llllll}
\hline Dependent variable & Independent variable & Gradient $(95 \%$ CI) & Intercept (95\% CI) & $R^{2}$ & Angle of attack range \\
\hline$C_{\mathrm{R}, \mathrm{FP}}$ & $C_{\mathrm{R}, 5 \text { areas }}$ & $0.964(0.924$ to 1.005$)$ & $-0.196(-0.280$ to -0.113$)$ & 0.991 & Full \\
$C_{\mathrm{R}, \mathrm{FP}}$ & $C_{\mathrm{R}, 5 \text { points }}$ & $0.945(0.877$ to 1.014$)$ & $-0.158(-0.262$ to -0.055$)$ & 0.983 & $-17^{\circ}$ to $63^{\circ}$ \\
$C_{\mathrm{R}, \mathrm{FP}}$ & $C_{\mathrm{R}, 5 \text { points }}$ & $1.688(0.695$ to 2.681$)$ & $-1.389(-3.772$ to 0.995$)$ & 0.793 & $68^{\circ}$ to $98^{\circ}$ \\
$C_{\mathrm{h}, \mathrm{FP}}$ & $C_{\mathrm{R}, 5 \text { areas }}$ & $0.826(0.788$ to 0.864$)$ & $-0.031(-0.039$ to 0.101$)$ & 0.989 & Full \\
$C_{\mathrm{v}, \mathrm{FP}}$ & $C_{\mathrm{R}, 5 \text { areas }}$ & $0.901(0.704$ to 1.098$)$ & $0.122(-0.063$ to 0.308$)$ & 0.803 & Full \\
$C_{\mathrm{h}, \mathrm{FP}}$ & $C_{\mathrm{R}, 5 \text { points }}$ & $1.022(0.902$ to 1.142$)$ & $-0.079(-0.263$ to 0.106$)$ & 0.934 & Full \\
$C_{\mathrm{v}, \mathrm{FP}}$ & $C_{\mathrm{R}, 5 \text { points }}$ & $0.818(0.634$ to 1.002$)$ & $0.193(0.015$ to 0.371$)$ & 0.795 & Full \\
\hline
\end{tabular}

$C_{\mathrm{v}}$ as a conservative, somewhat underestimating proxy to $C_{\mathrm{L}}$, the maximum lift of the revolving pigeon wing exceeds that of bird wings measured in the steady, translating flow of windtunnels. Maximum lift coefficients for a range of bird wings (Withers 1981) and a model pigeon wing (Nachtigall 1979) measured in windtunnels only achieve 1.2. Withers' bird wings achieve their maximum lift at angles of attack (at mid-wing) at $8^{\circ}-25^{\circ}$. These measurements contrast with the $C_{\mathrm{v}}$ of 1.49 or $C_{\mathrm{L}, \max }$ of 1.64 (or even higher, Fig. 3c, d) reported here for the revolving pigeon wing, at angles of attack of $38-43^{\circ}$ at the wingtip. The most parsimonious explanation for this, at least until near-field flow modelling or visualisation can be performed on pigeon wings, is that some aspect of 3-D flow is present in the revolving (propeller) case and absent in translating (windtunnel) case; and that this spanwise flow disrupts the process of conventional stall, resulting in high force coefficients at high angles of attack.

\subsection{Pressure maps}

The pressure map data for the real, dried pigeon wing revolving at $4 \mathrm{~Hz}$ is presented in two forms (Fig. 4, 5): as coefficients of pressure, which normalises point pressures by air density and local velocity (Fig. 4), and $k_{\mathrm{p}}$, that normalises by density and angular velocity (Fig. 5). Higher than wing-average coefficients of pressure are observed towards the base of the wing, consistent with their relatively thick and highly cambered aerofoil sections. In contrast, coefficients towards the wingtip are close to the average for the wing area. This is consistent with the dominant contribution of the aerodynamic forces of the distal wing to the overall wing (despite their lower coefficients) due to their much higher absolute velocities (Fig. 5).

Integrating the pressure maps over the wing area by two techniques appears largely effective in predicting net forces (Figs. 6, 7). By taking the average pressure as representative, or the pressure at the sensor near the midline of the wing (Fig. 1c), for each of five wing sections, the resultant aerodynamic force coefficients $\left(C_{\mathrm{R}, 5 \text { areas }}\right.$ and $C_{\mathrm{R}, 5 \text { points }}$ respectively) correlates well with that measured in the forceplate experiments $C_{\mathrm{R}, \mathrm{FP}}$, albeit with a slight offset in angle of attack. Linear regressions (Table 2) show that the areas method is reasonably accurate over the whole range of angles of attack, and the midline, five-points method for angles between $-17^{\circ}$ and $63^{\circ}$. At higher angles, midline-only measurements start to undervalue the resultant forces. Also, resolving the resultant force into vertical and horizontal components by assuming that the force on each section acts perpendicular to the wing chord is largely successful (Figs. 6c, d; 7c, d). Over all angles of attack, the linear regressions for horizontal and vertical force coefficient measurements derived from either pressure-based technique provide, depending on the level of accuracy required, a reasonable match with those calculated from the forceplate measurements (Table 2). These findings provide some support for the techniques of Usherwood et al. (2005), indicating that direct, local pressure measurements can be effective in determining the whole-wing lift and drag for slow, flapping flight. In that the midline-only measurements were, within limits, effective for determining wing forces, the feasibility of using a reduced array of pressure measurements to determine aerodynamic power requirements in flapping, free-flying birds is encouraging. Whether this technique is appropriate for higher speed flight, where attached flow and leading-edge suction are presumably maintained, and the assumption that the resultant force acts perpendicular to the wing chord potentially less valid, remains uncertain.

\subsection{Stall and mechanisms of high resultant forces}

The flow structure about a revolving pigeon wing cannot be determined without some form of flow visualisation. However, the pressure map, notably the variability in the 
measured signal, can be highly informative. A band of low pressure running lengthways along the wing near the leading edge, that would be consistent with the presence of a stable leading-edge vortex (e.g. Liu et al. 1998), is not observed. Whether this is due to the absence of a stable leading-edge vortex structure, or the limited spatial resolution of the sensors is unclear. However, given the success in determining net aerodynamic forces from summing the local pressures, it appears that invoking an unmeasured region of very high differential pressure due to a tight, stable leading-edge vortex is unnecessary. What is clear is that, at an angle of attack between $33^{\circ}$ and $38^{\circ}$, there is a dramatic increase in variability of the pressure signal across the wing (Figs. 4, 5). This occurs at approximately the angle of attack leading to maximum vertical force (or lift) (Figs. 6, 7), and is good evidence either for some form of stall, or aeroelastic ringing, or both. Given the higher lift coefficients, and higher angles of attack for maximum lift, of the revolving wings described here than the windtunnel measurements of Withers (1981), it appears reasonable to suppose that some aspect of revolution delays full, conventional stall.

\subsection{Limitations and relevance of propeller measurements to bird flight}

The process of removal, drying, mounting and spinning a dead bird wing clearly departs from the reality of live, dynamically (both actively and passively) controlled flapping wings in myriad ways, and the motivation behind developing direct pressure measurement techniques is to advance the study of birds in free, near-natural flight. While the main focus of this paper is in validating such techniques, it is worthwhile considering whether the measurements made might provide insight into the aerodynamics of real pigeon flight. Recent kinematic measurements of pigeons in slow flight (Berg and Biewener 2008) demonstrate that some of the conditions experienced by a real pigeon may be more closely simulated with a propeller setup than traditional windtunnel setup. Very high angles of attack (in the conventional pre-stall sense) were observed during mid downstroke: at the wrist during level flight, the angle of attack was $40^{\circ} \pm 1^{\circ}(\mathrm{SE})$. In addition, the lift and drag coefficients estimated from kinematics were $1.44 \pm 0.29$ and $1.01 \pm 0.08$, respectively. While such measurements are not in exact agreement with propeller-based measurements-at a tip angle of attack of $40^{\circ}$, the forceplatederived $C_{v}$ coefficient was between 1.49 and 1.48 , and $C_{\mathrm{h}}$ between 0.86 and 1.02 - they provide a considerably closer match than windtunnel measurements on real bird wings.
3.6 Profile drag and power calculations in slow bird flight

It is worth commenting here on the apparent discrepancy between the values of $C_{\mathrm{D} \text {,pro }}$ reported here for bird wings, and those reported elsewhere for bird wings (e.g. Rayner 1979b; Pennycuick et al. 1992). Historically, analyses of bird flight performance have made the convenient, and perhaps not unreasonable when considering flight at medium and high air speeds, assumption that profile drag coefficient should approximate the minimum or no-lift drag coefficient. At lower flight speeds, requiring higher angles of incidence, this is certainly not the case (e.g. Withers 1981; Pennycuick et al. 1992). Under these conditions, $C_{\mathrm{D} \text {,pro }}$ derived from low-drag configurations$C_{\mathrm{D}, \text { pro }}=0.02$ appears a widely accepted rule of thumb (e.g. Askew et al. 2001; Askew and Ellerby 2007)—must be inappropriate. Therefore, just as methods for calculating aerodynamic power in hovering insects have had to be revised (Ellington 1999) (resulting in dramatically higher values; see, for instance Fry et al. 2005) following flapper and propeller experiments leading to the rejection of Ellington's (though reasonable at the time) approximation for low Reynolds numbers of $C_{\mathrm{D} \text {,pro }}=7 / \sqrt{R e}$ (Ellington 1984c), so may calculations of power in low-speed bird flight. One further note of caution is worthwhile: when considering flight with strongly inclined stroke planes [typical of slow bird flight other than hummingbirds (see Norberg 1975; Berg and Biewener 2008)], profile drag will contribute to weight support, and so the traditional distinctions between induced and profile powers become confounded.

Acknowledgments This work was funded by The Wellcome Trust. Bret Tobalske and Ty Hedrick provided valuable criticism throughout this project.

Open Access This article is distributed under the terms of the Creative Commons Attribution Noncommercial License which permits any noncommercial use, distribution, and reproduction in any medium, provided the original author(s) and source are credited.

\section{References}

Altshuler DL, Dudley R, Ellington CP (2004) Aerodynamic forces of revolving hummingbird wings and wing models. J Zool Lond 264:145-181

Askew GN, Ellerby DJ (2007) The mechanical power requirements of avian flight. Biol Lett 3:445-448

Askew GN, Marsh RL (2001) The mechanical power output of the pectoralis muscle of blue-breasted quail (Coturnix chinensis): the in vivo length cycle and its implications for muscle performance. J Exp Biol 204:3587-3600 
Askew GN, Marsh RL, Ellington CP (2001) The mechanical power output of the flight muscles of the blue-breasted quail (Coturnis chinensis) during take-off. J Exp Biol 204:3601-3619

Berg AM, Biewener AA (2008) Kinematics and power requirements of ascending and descending flight in the pigeon (Columba livia). J Exp Biol 211:1120-1130

Dickinson MH (1996) Unsteady mechanisms of force generation in aquatic and aerial locomotion. Am Zool 36:536-554

Dickson WB, Dickinson MH (2004) The effect of advance ratio on the aerodynamics of revolving wings. J Exp Biol 207:4269-4281

Dickinson MH, Lehmann F-O, Sane SP (1999) Wing rotation and the aerodynamic basis of insect flight. Science 284:1954-1960

Ellington CP (1984a) The aerodynamics of hovering insect flight. I. The quasi-steady analysis. Phil Trans R Soc Lond B 305:1-15

Ellington CP (1984b) The aerodynamics of hovering insect flight. II. Morphological parameters. Phil Trans R Soc Lond B 305:17-40

Ellington CP (1984c) The aerodynamics of hovering insect flight. VI. Lift and power requirements. Phil Trans R Soc Lond B 305:145181

Ellington CP (1999) The novel aerodynamics of insect flight: applications to micro-air vehicles. J Exp Biol 202:3439-3448

Ellington CP (2006) Insects versus birds: the great divide (invited). In: Charles Ellington, 44th AIAA Aerospace Sciences Meeting and Exhibit, Reno, Nevada, 9-12 Jan 2006. AIAA-2006-35, University of Cambridge, Cambridge http://www.aiaa.org/ content.cfm?pageid $=406 \&$ gTable $=$ Paper $\&$ gID $=47786$

Ellington CP, Van den Berg C, Willmott AP, Thomas ALR (1996) Leading-edge vortices in insect flight. Nature 384:626-630

Fry SN, Sayaman R, Dickinson MH (2005) The aerodynamics of hovering flight in Drosophila. J Exp Biol 208:2303-2318

Hedrick TL, Daniel TL (2006) Flight control in the hawkmoth Manduca sexta: the inverse problem of hovering. J Exp Biol 209:3114-3130

Liu H, Ellington CP, Kawachi K, Van Den Berg C, Willmott A (1998) A computational fluid dynamic study of hawkmoth hovering. J Exp Biol 201:461-477

Nachtigall W (1979) Der Taubenflugel in Gleitflugstellung; Geometrische Kenngroflen der Flugelprofile und Luftkrafterzeugung. J Ornithol iao, 30-40

Norberg UM (1975) Hovering flight of the pied flycatcher (Ficedula hypoleuca) In: Wu TY, Brokaw CJ, Brennen C (eds) Swimming and flying in nature, vol 2. Plenum Press, New York, pp 869881

Osborne MFM (1951) Aerodynamics of flapping flight with application to insects. J Exp Biol 28:221-245

Pennycuick CJ (1975) Mechanics of flight. In: Farner DS, King JR (eds) Avian biology, vol 5. Academic Press, London, pp 1-75

Pennycuick CJ (1989) Bird flight performance: a practical calculation manual. Oxford University Press, Oxford

Pennycuick CJ, Heine CE, Kirkpatrick SJ, Fuller MR (1992) The profile drag coefficient of a Harris' hawk wing, measured by wake sampling in a wind tunnel. J Exp Biol 165:1-19

Rayner JMV (1979a) A vortex theory of animal flight. Part 2. The forward flight of birds. J Fluid Mech 91:731-763

Rayner JMV (1979b) A new approach to animal flight mechanics. J Exp Biol 80:17-54

Usherwood JR (2008) Collared doves Streptopelia decaocto display with high, near-maximal muscle powers, but at low energetic cost. J Avian Biol 39:19-23. doi:10.1111/j.2007.0908-8857. 04347.x

Usherwood JR, Ellington CP (2002a) The aerodynamics of revolving wings. I. Model hawkmoth wings. J Exp Biol 205:1547-1564

Usherwood JR, Ellington CP (2002b) The aerodynamics of revolving wings. II. Propeller force coefficients from mayfly to quail. J Exp Biol 205:1565-1576

Usherwood JR, Hedrick TL, Biewener AA (2003) The aerodynamics of avian take-off from direct pressure measurements in Canada geese (Branta canadensis). J Exp Biol 206:4051-4056

Usherwood JR, Hedrick TL, McGowan CP, Biewener AA (2005) Dynamic pressure maps for wings and tails of pigeons in slow, flapping flight, and their energetic implications. J Exp Biol 208:355-369

Van den Berg C, Rayner JMV (1995) The moment of inertia of bird wings and the inertial power requirement for flapping flight. $\mathrm{J}$ Exp Biol 198:1655-1664

Weis-Fogh T (1973) Quick estimates of flight fitness in hovering animals, including novel mechanisms for lift production. J Exp Biol 59:169-230

Withers PC (1981) An aerodynamic analysis of bird wings as fixed aerofoils. J Exp Biol 90:143-162 